\title{
Multi-methodological Approach to Evaluate Performances of Mucoadhesive Oral Gel Based on Nanoparticles
}

\author{
P. Capra ${ }^{1}$, M. Bleve ${ }^{2}$, G. Musitelli ${ }^{1}$, F. Pavanetto ${ }^{1}$ and P. Perugini $1^{2}$ \\ 1. Department of Drug Sciences, University of Pavia, Via Taramelli 12, 27100 Pavia \\ 2. Etichub s.r.l., Department of Drug Sciences, Via Taramelli 12, 27100 Pavia.
}

\begin{abstract}
Oral mucositis due to chemotherapy and irradiation continues to be an important clinical problem. The effectiveness of hyaluronic acid-based compounds in accelerated healing and helping manage pain in patients with oral mucositis was demonstrated. It was investigated a protective and regenerative effect of hyaluronic acid based gel formulation enriched with NAG (N-acetyl glucosamine) loaded solid lipid nanoparticles against degenerative process of the oral mucosa. Gel formulations were obtained by adding sHA (sodium hyaluronate) into SLN aqueous suspension. Gel performances were evaluated by multi-methodological approach: mucoadhesive and barrier properties evaluation, cell viability. It was shown that gel formulation based sHA, enriched with NAG loaded SLNs, when added as suspension, demonstrated to have a good mucoadhesion profile comparable in terms of tensile work and fracture strength to Carbomer 934 2\%. The presence of NAG encapsulated and not in gel formulation enhances also the biocompatibility of the system, demonstrating also to have a proliferative effect. Finally, any barrier property was altered. Finally, results demonstrated that sHA based gel formulation enriched SLNs, demonstrated good mucoadeshion property, comparable to carbopol gel, positive control. The proposed gel formulation enriched with SLNs setting up in this work could be used as innovation strategy to treat oral mucositis.
\end{abstract}

Key words: Solid lipid nanoparticle, N-Acetyl Glucosamine, Oral injuries, Mucoadesion, In vitro TEWL (trans epidermal water loss) evaluation.

\section{Introduction}

Oral mucositis due to cancer therapies such as high-dose chemotherapy and/or radiation continues to be an important clinical problems [1]. Mucositis is an inflammatory and/or ulcerative lesions of the oral and gastrointestinal tract. It can manifest as erithema, atrophy, erosions, and ulcers and it is frequently accompanied by severe pain requiring narcotic analgesia. Increased severity of oral mucositis is associated with fever, risk of infection, dependency on total parenteral nutrition, use of intravenous analgesics, and mortality within the first 100 days [2-4]. Infectious disease, immune deficiency and medications can be causative. However, one of the major causes of mucositis is high dose cancer therapy. The oral pain,

Corresponding author: P. Capra, Department of Drug Sciences, University of Pavia, Via Taramelli 12, 27100 Pavia. also associated with the lesions frequently leads to the need for parenteral nutritional support with or without use of a feeding tube or gastrostomy, as well as use of opioids. Today, according to basic oral care and good clinical practice, many different therapeutic approaches are used and applied to patients: non medicated oral rinses (e.g. saline mouth rinses 4-6 times/day), use of a soft toothbrush, topical anesthetics, morphine administration for pain, benzydamine and chlorhexidine oral rinse, oral cryotherapy and many other strategies [5, 6].

New therapeutic or preventive mechanism-based strategies are urgently required. Transdermal and transmucosal drug delivery offer attractive alternative routes for administration for drugs and may avoid the significant drawbacks of peroral and parenteral administration. These routes of administration bypass 
first-pass metabolism by delivering drug directly into the systemic circulation. Low permeability due to skin's stratum corneum limits the feasibility of transdermal delivery of pharmacological agents, leaving transmucosal routes with greater permeabilities as the only significant alternative. In particular, the buccal mucosa, compared to the other routes of administration, has a larger surface area for drug application, better patient compliance, ease of dosage form removal in emergencies, robustness, and good accessibility [7].

In this context, the objective of this investigation was to develop a mucoadhesive gel formulation containing NAG (N-acetylglucosamine) loaded SLNs (solid lipid nanoparticles) and to investigate their efficacy and tolerability.

SLNs (solid lipid nanoparticles) are alternatives nano-system to polymeric nanoparticles, liposomes and other colloidal systems. They can formulate to load lipophilic or hydrophilic drugs. Advantages of SLNs include the composition (physiological compounds), rapid and effective production process including the possibility of large scale production, the avoidance of organic solvents and the possibility to produce high concentrate suspensions. Disadvantages include low drug-loading capacities, the presence of alternative colloidal structures (micelles, liposomes, mixed micelles, drug nanocrystals), the complexity of the physical state of the lipid $[8,9]$.

$\mathrm{N}$-acetyl-glucosamine is a component of hyaluronic acid, naturally occurring polyanionic, polysaccharide $[10,11]$. Hyaluronic acid is present in the intercellular matrix of most vertebrate connective tissues especially skin where it has a protective, structure stabilizing and shock-absorbing role. HA (hyaluronic acid) exists in vivo as a polyanion and not in the protonated acid form. It is ubiquitous and it is distributed widely in vertebrates and present as a component of the cell coat of many strains of bacteria [12-14]. Extensive studies about chemical and physicochemical properties of HA and its physiological role in humans, together several properties, such as biocompatibility, biodegradability, non-immunogenicity and viscoelasticity, have proved that is an ideal biomaterial for cosmetic, medical and pharmaceutical applications. Its properties are dependent from commercially available molecular weights.

The use of HA in the treatment of oral mucositis is known from many years. In 2001, the FDA approved a gel based on sodium hyaluronate as a Class I medical device for use in the management of pain relief and useful in patients affected from oral mucositis. More recently, Cirillo et al. [14] demonstrated that Aminogam ${ }^{\circledR}$, compound containing a pool of aminoacids and sodium hyaluronate accelerated healing and helped manage pain of patients with oral mucositis [15]. Finally, studies about polyvinylpirrolidone-sodium hyaluronate preparations in the form of bioadherent gel (Gelclair®) suggest that the compound acts as a merely physical barrier between the oral environment and oral mucosa, reducing pain and promoting healing [16].

Finally, the aim of this work was to demonstrate the capability of gel enriched with NAG loaded SLNs in terms of mucoadhesion, bioavailability and epithelium integrity property.

\section{Materials and Methods}

\subsection{Materials}

N-Acetyl-d-glucosamine (NAG, purity $>99 \%$, CAS number 7512-17-6) was purchased from Sigma-Aldrich (Milan, Italy), and stored at $-20{ }^{\circ} \mathrm{C}$ in the dark until use as recommended by the manufacturer. Myristyl Myristate and Cetearyl Glucoside were obtained from Evonik Industries, Italy. Lipoid E 80 (purified phosphatidylcholine from egg lecithin) was generously gifted by Lipoid $\mathrm{GmbH}$, Ludwigshafen, Germany. sHA (sodium hyluronate) was obtained from Giusto Faravelli (Milan, Italy). The cross-linked polymer used was Carbopol 934P, (cross-linked (poly (acrylic acid, Lubrizol, USA). Ultra 
pure water was used throughout the experiments.

\subsection{Methods}

\subsubsection{Preparation of SLNs}

NAG loaded SLNs were prepared by hot melted lipid modified homogenization. Production parameters such as mixing and speed time, total formulation volume and lipid/water ratio were previously set up [17, 18]. Briefly, Myrystil Myrystate and Cetearyl Glucosid as lipid phase, NAG and egg lecithin as the aqueous phase in filtered water were heated to $85^{\circ} \mathrm{C}$, separately. Then aqueous phase was added to lipid phase maintaining a speed at $11,000 \mathrm{rpm}$ for 2 minutes homogenizing by Silverson 2ST (Silverson, United States).

The obtained emulsion $\mathrm{O} / \mathrm{W}$ emulsion was homogenized at 11,000 for 1 minute in an ice bath and maintained for 1 hour at low temperature (about $0-2{ }^{\circ} \mathrm{C}$ ) in order to recrystallize the lipid phase to the solid state in the form of a SLN aqueous suspension. Drug loaded SLNs were prepared by adding NAG in the internal aqueous phase before the addition of lipid phase to the external aqueous phase.

Placebo and loaded SLNs were characterized previously by evaluating particle size, drug loaded efficiency (EE\%), calorimetry profile (data not reported).

Gradient centrifugation was used like a purification method to eliminate free NAG (not encapsulated). A $20 \%$ $\mathrm{NaCl}$ solution was put in a microtubes and SLN suspension was placed on the top. Samples were centrifuged for 5 minutes at $21.700 \mathrm{rcf}, 16^{\circ} \mathrm{C}$ (Thermo Scientific SL 8R Centrifuge). After centrifuge the suspension was recovered.

\subsubsection{Preparation of Gels Containing NAG Loaded SLNs}

Gel was prepared by dispersing sodium hyaluronate $(0.2 \%)$ in water at $40{ }^{\circ} \mathrm{C}$ and mechanically stirring until room temperature was reached. Free NAG or NAG-loaded SLNs were then dispersed in the final preparation under gentle stirring. In Table 2 quali-quantitative of setting up gel formulations is reported.

Carbopol 934 based gel (2\%) was also prepared as a positive sample control, as reported in literature [19-21]. Briefly, polymer was dispersed in water and leaved overnight in order to polymer hydration and, finally, led to $\mathrm{pH} 4.5$ with sodium hydroxide. As a negative control was used an artificial saliva solution for which was demonstrated any interaction with mucosal tissue.

\subsubsection{Evaluation of Mucoadhesive Properties}

The mucoadhesion measurements were performed using the AGS 500ND tensile machine (Shimadzu corporation Kyoto-Japan) equipped with a $10 \mathrm{~g}(0.1 \mathrm{~N})$ load cell, a modified conical probe and bioadhesive support. A mucin tablet with surface area of $254 \mathrm{~mm}^{2}$ was anchored to load cell and imbibed with 5\% mucin solution (mucin type II crude, Sigma-Aldrich). The formulations were transferred into modified multi-plate $(5 \mathrm{~g})$ maintained at $37{ }^{\circ} \mathrm{C} \pm 0.5{ }^{\circ} \mathrm{C}$ in a water bath. Samples and substrate were put in contact with a preload of $4 \mathrm{mN} / \mathrm{cm}^{2}$ for 30 seconds. The probe

Table 1 Composition (\% w/w) of placebo and loaded SLNs.

\begin{tabular}{lll}
\hline INCI name & SLN $_{\mathrm{p}}$ & SLN $_{1}$ \\
\hline Myrystil myrystate & 3.6 & 3.6 \\
Cetaryl Glucoside & 1.2 & 1.2 \\
Phosphatidylcholine & 1.2 & 1.2 \\
Aqua & 94 & 92.8 \\
NAG & - & 1.2 \\
\hline
\end{tabular}

Table 2 Quali-quantitative composition of setting up gel formulation.

\begin{tabular}{lll}
\hline Batch & SLN Ingredients & Percentage \\
\hline A-Hydrogel & - & - \\
B-SLN placebo & Cetearyl Glucoside & 0.6 \\
& Myristyl Myristate & 1.8 \\
& Cetearyl Glucoside & 0.6 \\
C-NAG-SLN ** & Myristyl Myristate & 1.8 \\
& NAG & 0.3 \\
& Cetearyl Glucoside & 0.6 \\
D-NAG-SLN *** & Myristyl Myristate & 1.8 \\
& NAG & 0.6 \\
E-NAG-Hydrogel & NAG solution & 0.6
\end{tabular}

*A-Hydrogel and E-NAG-Hydrogel represents control batches, without SLNs. 
**C-NAG-SLN batch is enriched with purified SLNs, obtained with gradient centrifugation.

***D-NAG-SLN batch is enriched with suspension SLNs.

was moved upwards at a predetermined speed at 10 $\mathrm{mm} / \mathrm{min}$ to the complete separation of the mucoadhesive interface (mucin-sample). Maximum detachment force was obtained from the force-distance graph. The area under the curve as the mucoadhesive took place was calculated from the force distance-plot. Each experiment was carried out three times.

2.2.4 In vitro MTT Assay by Reconstituted Human Oral Model

Assessment of the activity of living cells, based on mithocondrial function, was determined according to OECD guidelines irritation test $n^{\circ} 439$ [22].

\subsection{Media and Reagents}

The RHO culture, maintenance and growth medium were all supplied by Episkin Laboratories (Nice, France). A sterile physiological solution $(\mathrm{NaCl} 0.9 \%$, solution A) and phosphate buffer solution $\mathrm{pH} 7.8$ (solution B) were prepared.

\subsection{Control Samples}

Negative control (solution A) and positive control $(0.5 \%$ w/v sodium dodecyl sulphate, SDS, Sigma-Aldrich) cultures were run in each assay to provide the necessary data for defining possible acceptance criteria.

\subsection{The Reconstituted Human Oral Epithelium}

To evaluate irritating potential of setting up samples, the RHO (reconstituted human oral epithelium) model from Episkin Laboratories was used. Immortalized human oral epithelial cells were cultured on an inert 0.5 $\mathrm{cm}^{2}$ polycarbonate filter at the air liquid interface in chemically defined medium [23] (Fig. 1). All tissue were manufactured under high-quality condition (GMP-like) and each batch of tissue is quality assured according to specific QC standards. A Quality Control Certificate was provided with every shipment of tissue. The inserts containing the human epithelium cultures were shipped in a multiwall plate filled with an agarose-nutrient solution in which they were embedded.

\subsection{Culture Preparation}

After arrival, RHO cultures were placed in $300 \mu \mathrm{l}$ fresh maintenance medium (24-well plate) and, after the absence of air bubbles underneath the culture inserts was verified, incubated (overnight) at $37^{\circ} \mathrm{C}, 5 \%$ $\mathrm{CO}_{2}$ in a humidified incubator. Following this equilibration period, the cultures were transferred into a new 24-well plate (containing $300 \mu$ Episkin maintenance medium in each well) for treatment. Since the nutrients in the medium were supplied through the basal cell layers, the surface of the oral epithelium could be treated topically with the test sample.

\subsection{Culture Treatment and Viability Determination}

Duplicate in vitro culture $0.5 \mathrm{~cm}^{2}$ were placed in 300 $\mu \mathrm{l}$ fresh maintenance medium and dosed topically with $50 \mathrm{mg}$ of semisolid formulations (gels) for 30 minutes before of irritative stress with SLS 5\% (30 $\mu$ l) for 15 minutes. Each sample was administered carefully onto the epithelial surface so that the complete area was covered. At the end of the treatment with SLS, each tissue was washed with phosphate buffer solution and the sample was re-applied. Two different incubation time were considered: 2 and 24 hours. Cultures treated with only SLS 5\% was considered irritation control. 


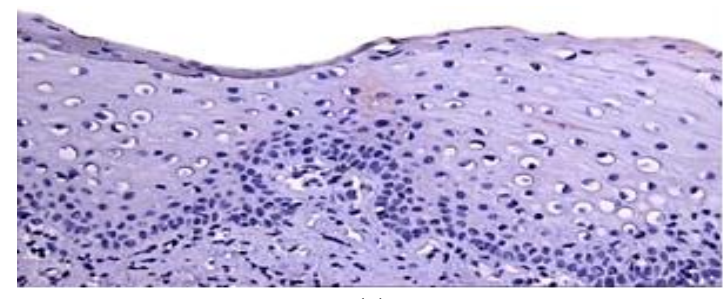

(a)

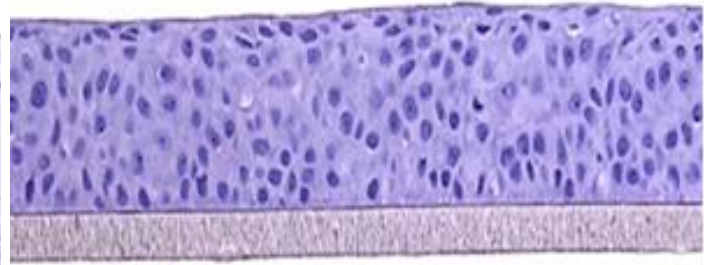

(b)

Fig. 1 In vivo mucosa of the oral cavity (a); Reconstructed Human Oral Epithelium by Episkin (Nice, France) (b).

Cultures treated with the samples without SLS solution application were considered as internal control of the experiment. Negative control (tissue alone) and positive control $(5 \% \mathrm{w} / \mathrm{v}$ SDS $)$ treated tissues were included in each experiment. At each time point, tissues were assessed for tissue viability (MTT assay). Following incubation, tissues were carefully rinsed with solution B and transferred to a freshly prepared MTT solution $(1 \mathrm{mg} / \mathrm{ml} ; 300 \mu \mathrm{l})$ in 24 -well plates. MTT is a yellow tetrazolium dye (3-(4,5-dimethylthiazol-2-yl)-2,5-diphenyltetrazolium bromide) that is reduced to a purple formazan by mitochondrial succinate dehydrogenase in viable cells [24]. The plates were then incubated for $3 \mathrm{~h}$ at $37^{\circ} \mathrm{C}$ in a humidified atmosphere of $5 \% \mathrm{CO}_{2}$ in air. The RHO tissues were removed from the MTT solution and immediately transferred to a 24-well plate containing isopropanol (750 $\mu \mathrm{l}$ per well). An additional $750 \mu \mathrm{l}$ isopropanol was added onto each tissue. The plates were gently shaken for at least 2 hours at room temperature to extract the reduced MTT (formazan crystals) out of the tissue. Finally, $100 \mu 1$ aliquots of the extracted MTT solution were transferred to a flat-bottom 96-well plate and the OD (optical density) of samples was measured at $595 \mathrm{~nm}$ with a microtiter plate reader (BIO RAD Model 550). The percentage viability of samples was calculated from the percentage MTT conversion in the sample treated cultures relative to the corresponding negative control (100\% viable).

Results were expressed as cell viability percentage according to following equation (Eq. 1)

Cell viability percentage $=[\mathrm{OD}$ sample/OD negative control] X 100

\subsection{Evaluation of Epithelium Integrity}

In order to evaluate the epithelium integrity, a method commonly used in vivo to evaluate the integrity of the barrier function of the skin, was employed. In fact, the measurement of the water loss from epithelium by the evaporimeter can give informations regarding the integrity of the tissue.

The test was performed by using Evaporimeter Tewameter TM 300 (Cutometer MPA580, Courage \& Khazaka, Cologne, Germany). This apparatus is equipped with probe with sensors that measure the humidity. In vitro measurements of water loss were conducted by directly placing the probe (open chamber) onto RHO cultures. During the test, RHO cultures were maintained at a constant temperature and relative humidity. The water flux values, expressed in $\mathrm{g} / \mathrm{h} / \mathrm{m} 2$, were taken at four different times: 15, 30, 60 minutes. Physiological solution was considered as a negative control. In order to reproduce in vivo condition, gel formulations were diluted with physiological solution according to ratio $1: 2$.

\section{Results and Discussion}

Solid lipid nanoparticles are colloidal carrier systems characterized from lipid matrix made of physiological lipids that decreases the danger of acute and chronic toxicity respect to polimeryc nanoparticles [25]. Moreover, the potential of SLNs in epidermal targeting, controlled release, negligible skin irritation, and protection of active compounds has well established.

In addition, the small particle size of SLNs ensures that the nanoparticles are in closed contact with 
mucosal tissue, thus promoting the amount of the encapsulated agent which penetrates into the skin/mucosa.

The aim of this work was to demonstrate the capability of gel enriched with NAG loaded SLNs in terms of mucoadhesion, bioavailability and epithelium integrity property. In fact, the key issues in the development of an oral mucosal products are toxicity, bioavailability (absorption and distribution), and metabolization of their components. In previous studies, physico-chemical characterization of SLNs and set up methods were investigated in order to demonstrate optimal dimensions, drug efficiency into the nanoparticles and its efficacy, the tolerability of each bulk materials and drug when applied onto keratinocytes $[18,26]$.

\subsection{Mucoadhesion Property}

Bioadhesion, or mucoadhesion, represents the ability of a biological or synthetic material "to stick" to a mucose membrane $[19,27,28]$. The result of this interaction is the adhesion of the material/product to tissue for prolonged time. For this reason bioadhesive formulation can be considered a drug delivery system characterized from enhanced drug bioavailability and prolonged pharmacological activity in the time.

In particular, a gel formulation for buccal application should exhibit high mucoadhesiveness to be able to fix the formulation on the buccal mucosa, allowing enough time for prolonged drug release, due to the appropriate mechanical properties.

In this study it was chosen to use a small volume of gel (5 g) with respect to the in vivo situation where it had a small gel volume in relation to large area of mucosa.

During preliminary studies, a low time of contact between mucin tablet and sample was established because the force acting on the probe gradually reduces and is approximately zero within 2 min [20]. However, Carbopol 934, used as positive control in this study, according to Hagerstrom et al., should show only a small decrease of the force over the time. For this reason Carbopol 934 2\% was chosen as standard. Moreover, if the measurements are performed at very long contact times, the risk of dehydratation of gel formulation is possible. Therefore, according to small volume of sample and relaxation phenomena, 30 "' was the contact time chosen.

Since surface and diffusion phenomena, interpenetration, formation of molecular entanglements and secondary chemical bonds are of great importance in the mucoadhesion process, mucin substrate (mucin tablet) was prehydratated for some seconds in order to enhance the mobility of the polymer chains and to enable rapid interactions with mucin substrate.

Table 3 reported for each single gel formulations mucoadhesion work $(\mathrm{Lm})$ and release pression $(\sigma)$. The first parameter represents the area under the force-distance curve and is function of release pressure and deformation. Instead, the second parameter represents the force per unit area of gel substrate that is required to release it from mucin tablet. Results were elaborated according to the following formula:

$$
\sigma=\mathrm{F}(\mathrm{t}) / \mathrm{S}
$$

Where $S$ is the interaction area between sample and mucin substrate and $\mathrm{F}(\mathrm{t})$ is the force applied for release (value reported from instrument) [21].

As shown in Table 3, the adhesion work of Batch A-Hydrogel differs significantly from the other samples, registering higher value. This was due to pronounced viscoelastic profile of sodium hyaluronate that was correlated to registered large deformation to failure (Fig. 3). Instead, Carbopol 934 2\% showed a smaller deformation to failure, as also confirmed from literature, leading to a relatively low tensile work though the fracture strength was as higher as Batch A-Hydrogel. For control and A-hydrogel batches, the significant mucoadhesion property was determined from chemical bonds, as hydrogen bonds, thanks the presence into molecular structure of carboxylic, hydroxyl and amino groups. For gel formulations 
Table 3 Measurements of mucoadhesive properties of gel formulations expressed as tensile work and fracture strength.

\begin{tabular}{llll}
\hline Batches & $\begin{array}{l}\text { Work of adhesion } \\
\text { Lm }\left(\mathrm{mN}^{*} \mathrm{~mm} \pm \text { S.D. }\right)\end{array}$ & $\begin{array}{l}\text { Maximum strenght } \\
\text { Fmax }(\mathrm{mN} \pm \text { S.D. })\end{array}$ & $\begin{array}{l}\text { Fmax/Area } \\
\sigma\left(\mathrm{mN} / \mathrm{cm}^{2}\right)\end{array}$ \\
\hline Carbomer 934 2\% & $110 \pm 10$ & $44 \pm 1$ & 19 \\
A-Hydrogel & $290 \pm 60$ & $44 \pm 1$ & 19 \\
B-SLN placebo & $90 \pm 10$ & $23 \pm 3$ & 10 \\
C-NAG-SLN & $80 \pm 10$ & $13 \pm 1$ & 6 \\
D-NAG-SLN & $100 \pm 10$ & $35 \pm 3$ & 15 \\
E-NAG-Hydrogel & $140 \pm 10$ & $28 \pm 1$ & 12 \\
\hline
\end{tabular}

*Artificial saliva, a negative control, confirmed close to zero, proving a sample completely inert towards the mucin substrate.

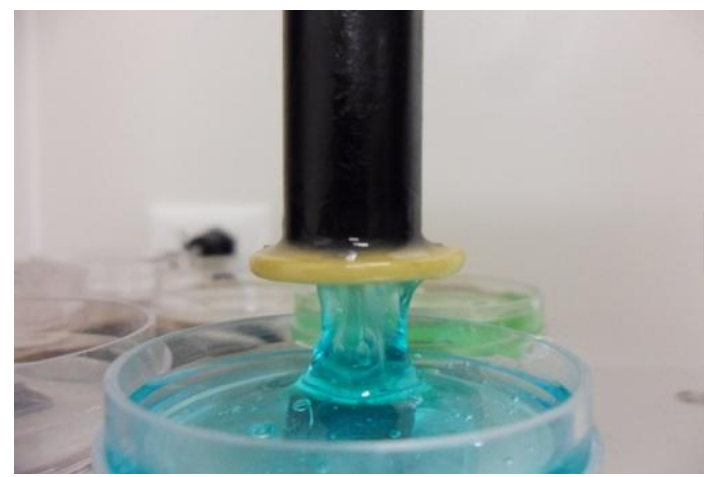

Fig. 2 Mucoadhesive test of hydrogel enriched with $\mathrm{SLN}_{\mathrm{l}}$.

enriched with nanoparticles was observed different profiles in terms of fracture strength, tensile work and deformation to failure. In particular, it was found a value reduction that could be explained from the lipid composition of SLNs, placebo and loaded, and from the presence of the surfactant. In according to literature hydrophilic polymer, as the same sodium hyaluronate and carbomer 934 used in this work, exhibit strong mucoadhesive property for the presence of polar groups. Instead, the lipid matrix of SLNs reduces the affinity for aqueous solution and so for mucin solution, with which was soaked mucin tablet, and consequently it reduces mucin tablet/gel formulation interactions. Moreover, surfactant could be cause of charge repulsion. Data reported in Table 3 show as the only batch with a good profile is D-NAG-SLN, enriched with a suspension of SLN, characterized from the presence of encapsulated and free NAG in the gel formulation. This could explain the better profile: the molecular structure of NAG and its polar groups improve interactions and consequently mucoadhesion profile. A similar tendency was determined also for E-NAG hydrogel batch confirming the role of NAG. On the contrary Batch C, enriched with a purified SLNs, showed a bad profile, probably characterized from a different organization of the lipid and surfactant into SLN matrix.

Last consideration, if the maximum force value demonstrates the existence of strong interactions between sample and substrate, percentage deformation (Fig. 3) shows the presence of weak bonds, which can be due to a dynamic ion exchange between sample and substrate. This could explain the differences reported in Fig. 3. According to this, maximum strength and deformation profiles are independent parameters.

In conclusion, Batch D-NAG-SLN demonstrated to have a good mucoadhesion profile, comparable to Carbomer 934 2\% one. 


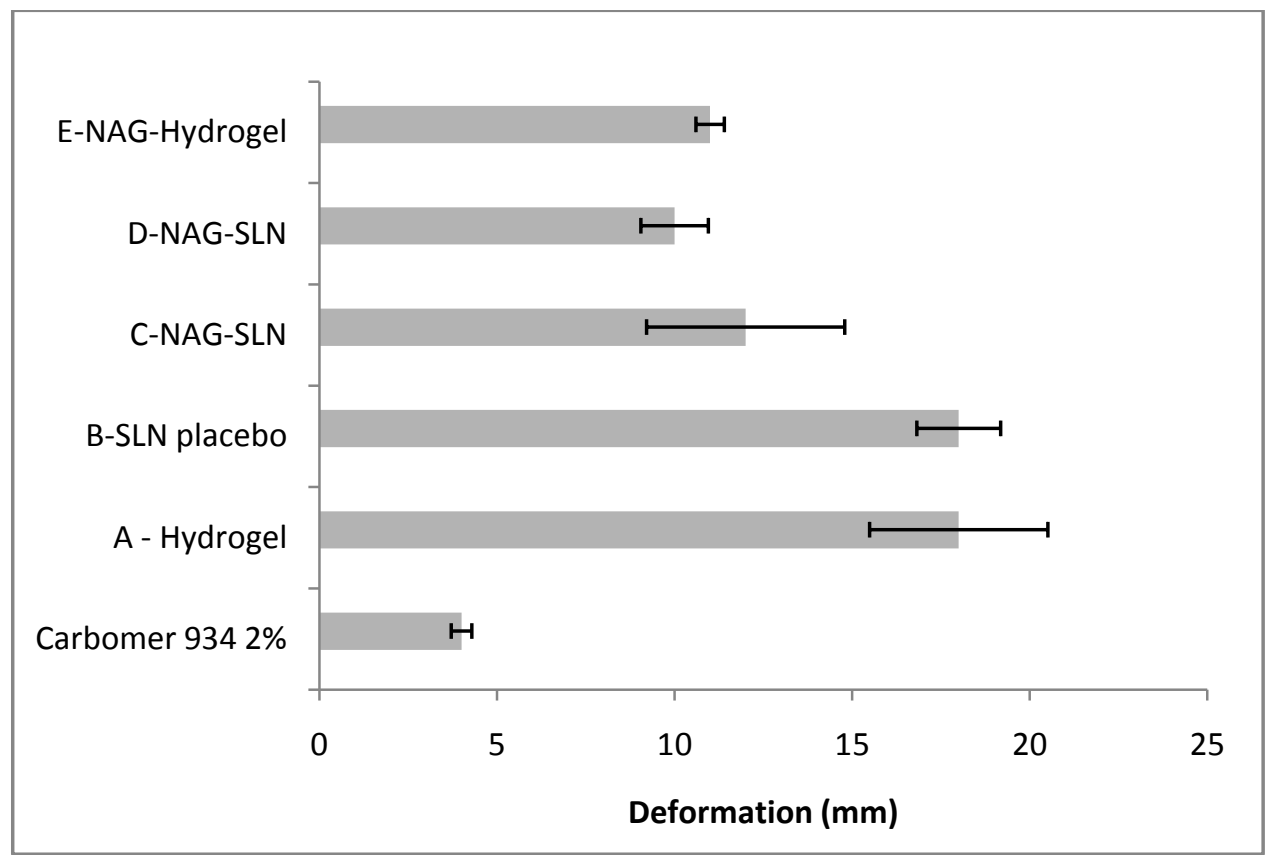

Fig. 3 Deformation to failure from mucoadhesion measurements of the samples.

\subsection{Cell Viability Determination of Samples after} Irritation Stress

Since the oral epithelium provides an important barrier function and is the first point of contact when substance or product enters the oral cavity, several in vitro models were developed to assess the effects of the same substance/product on multi layered epithelial tissues. In this work reconstituted oral human tissue were used in order to verify the biocompatibility and barrier properties of the reconstituted tissue when they are stressed from an irritant substance as SDS. The first critical point of the study it was the high viscosity of the gel formulation that which made it difficult for its removal from the tissue for the subsequent application of the MTT solution. More and more washes were necessary in order to remove completely products from culture and also this could compromise the survival of the cells.

In a previous study the tolerability of SLN formulation and their individual components was evaluated [23]. The evaluation of EC50 parameter of single each sample (bulk material and SLNs) allowed the choice of the best quali-quantitative composition in order to obtain a safe and well-tolerated product. The presence of surfactant in the SLN preparation could reduce the tolerability of the nanoparticles, and so, in order to evaluate the influence of SLN and the presence of the active, EC50 approach was used; the EC50 values were calculated using linear regression analysis of the data. In detail, according to EC50\% of lipid matrix, estimated at $0.018 \%$, a same concentration into SLNs was applied onto reconstituted tissue. As it is possible to observe in Fig. 4, after $2 \mathrm{~h}$ of incubation all gel formulations maintain a good biocompatibility profile. From Batch A to Batch D comparable profiles were observed: for all batches a cell viability percentage of about $80 \%$ was measured.

In particular, Batch $\mathrm{C}$ and $\mathrm{D}$, containing NAG loaded SLNs, show a very good profile until $24 \mathrm{~h}$ of contact, respect Batch A and B; NAG, according to previous study, demonstrated significant proliferative properties (Fig. 4). After 24 hours of sample contact, cell viability reduction may be due to the strong adhesion of the gel to the tissue, which after many hours of incubation could hinder the gaseous interchanges between cells and environment.

Protective effect of SLN batches, at $2 \mathrm{~h}$ of incubation, 
was confirmed also using higher lipid concentrations.

All batches maintained a cell viability of over $85 \%$

(Fig. 5). This was also predictable considering the fact that the lipids used for the SLN setting up were physiological lipids that constituted the skin hydro-lipid film.

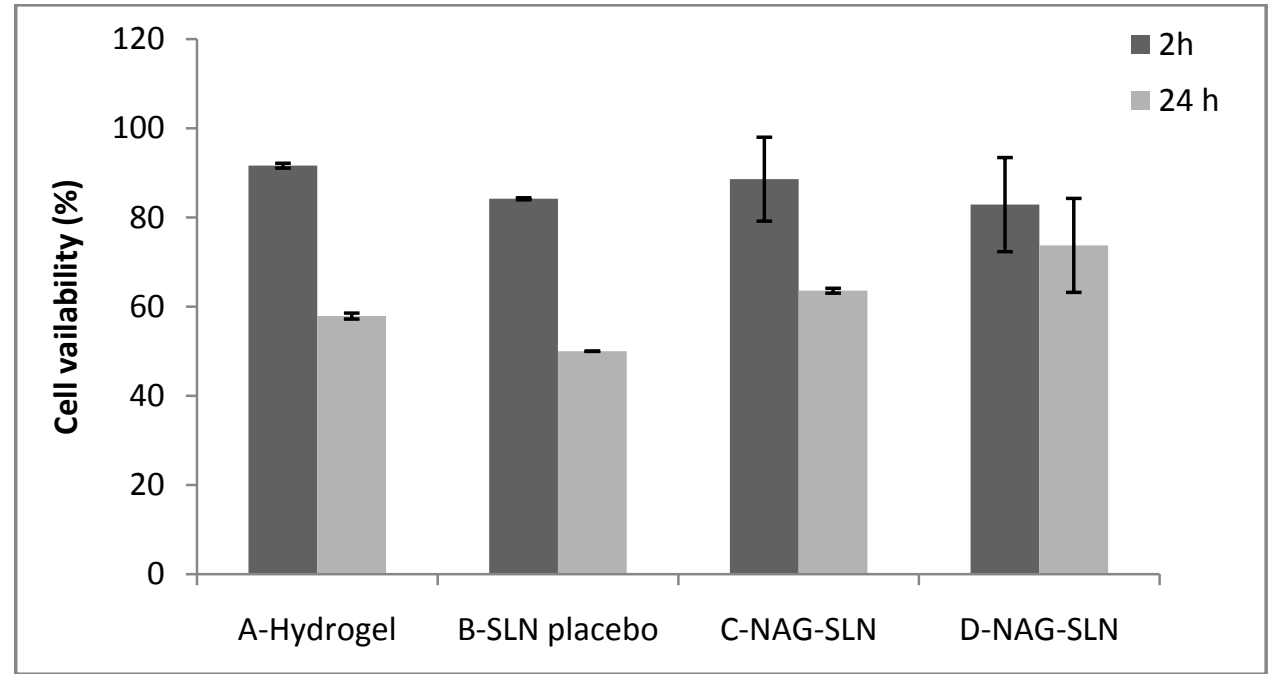

Fig. 4 Cell viability (\%) of gel formulations over time. The percentage viability of samples was calculated respect to negative control (100\% viable) (data not reported).

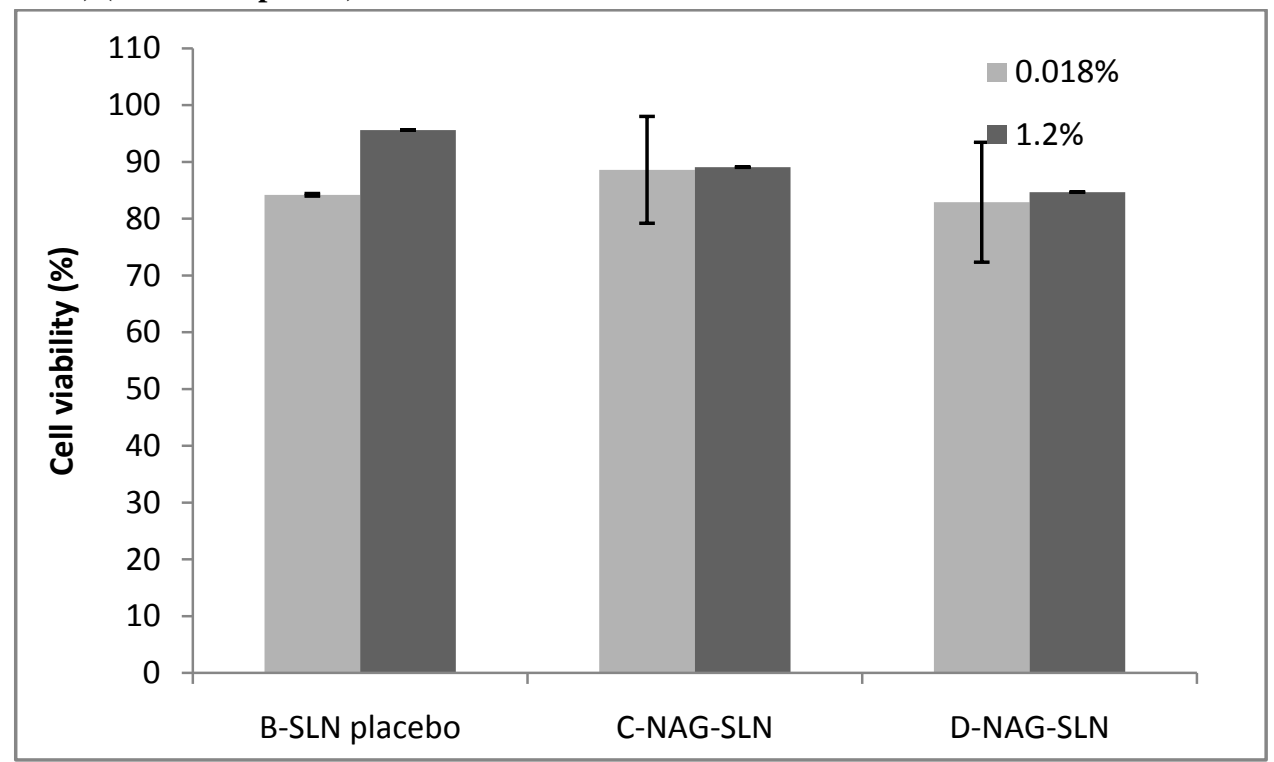

Fig. 5 Cell viability (\%) of gel formulations enriched with placebo and loaded SLNs at different lipid concentrations. 


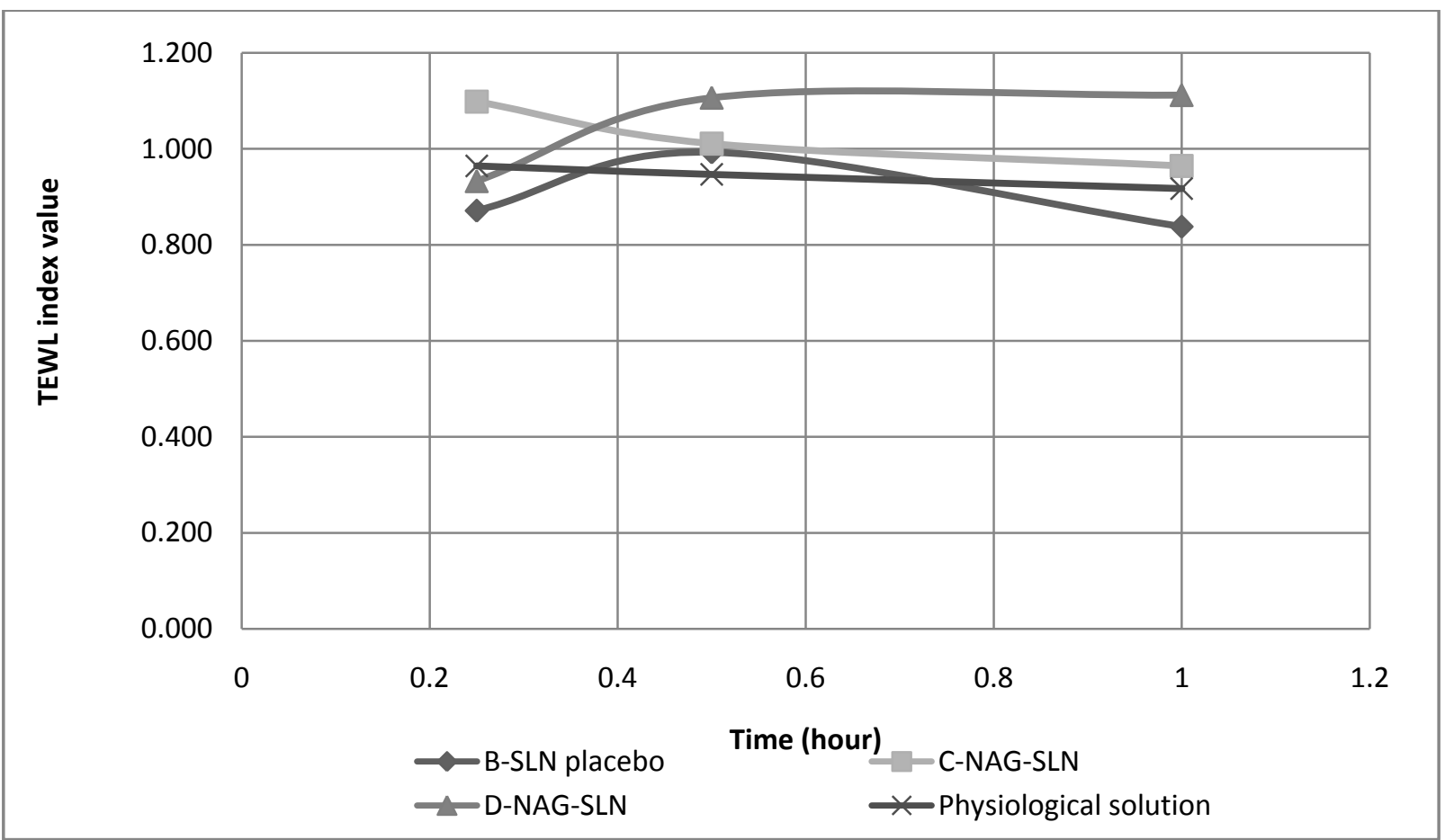

Fig. 6 TEWL data, reported as index value, of gel formulation enriched with and without placebo and loaded SLNs.

\subsection{TEWL Evaluation of Gel Formulation}

The purpose of this test was to evaluate the barrier properties of the epithelium after application of the compound.

TEWL measurements demonstrated to give a good correlation with other in vivo methods for determining skin tolerability, such as visual scoring and colorimetry. In particular, following irritation, a decrease in barrier function can occur, which in turn is reflected by an increase in TEWL. Theoretically, the more irritating a compound is, the greater increase on TEWL value will be observed.

Generally, in vivo TEWL measurements are conducted by directly placing the TEWL probe onto the skin. Steady-state conditions are reached when the standard deviation values remain low. This is usually achieved in a few seconds (25"). Instead, for in vitro studies, the TEWL probe cannot be directly placed onto the tissue surface, but rather it lies on top of the RHO support. The increased distance between the skin surface and the sensor causes the time to steady state to increase up to a few minutes. For this reason an adapter was created which resulted in a better seal between the diffusion cell and the TEWL probe in order to improve the time to and achievement of steady-state in vitro conditions.

Data acquired from experiments are shown in Fig. 6. TEWL index baseline of negative control confirmed the integrity maintenance of the RHO during the test, demonstrating stable and reproducible over time. In particular, the value was considered comparable to healthy skin one. The values for Batches B, C and D were also comparable over time. In particular, TEWL index values, ranging around 1 , confirmed that reconstituted tissue structure was not affected by composition of the product, maintaining its barrier properties. On the contrary, the profile of Batch A was completely different and not comparable with which of the other ones. The low values measured were maintained over time. This results highlighted that the molecular structure of the hydrogel composing batch A avoid the water evaporation strongly interacting with the tissue, in according with previously reported mucoadhesion data. This behavior was also experimentally demonstrated because batch A- 
hydrogel was removed from tissue only after more and more washing cycles.

\section{Conclusions}

A mucoadhesive and protective gel enriched with NAG loaded SLNs was successfully prepared in the work here described.

The batch D-NAG-SLN, containing NAG inside and outside SLNs, demonstrated a good mucoadhesive property, any irritant effect onto in vitro cultures, demonstrating its protective effect and its capability to guarantee the maintenance of good barrier properties of the mucosa tissue. The novelty of this work was also to use the RHO (reconstructed human oral epithelium) not only to verify cell viability and the tolerability of the applied product but also to verify barrier properties over the time by evaluation of the water lost through the epitelium never done before.

The last aim of this study was to set up a protocol in order to quickly evaluate the performances of products for oral treatment, before to apply the product onto patients.

Finally, the study protocol developed in this project can be considered a valid approach to investigate new products as it allows the pre-screening of the product in terms of safety and efficacy, ensuring immediate and reproducible results.

\section{References}

[1] Peterson, D. E., Bensadoun, R. J., and Roila, F. 2011. "Management of Oral and Gastrointestinal Mucositis: ESMO Clinical Practice Guidelines.” Annals Oncology 22 (6): v78-v84;

[2] Elad, S., and Thierer, T. "Cost-effective Analysis of Topical Chlorexidine in Hematologic Patients at Risk for Oral Mucositis." Clin. Oral Invest., DOI 10.1007/s00784-015-1438-z).

[3] Sonis, S. T., Oster, G., Fuchus, H., Bellm, L., Bradford, W. Z., Edelsberg, J., Hayden, V., Eilers, J., Epstein, J. B., LeVeque, F. G., Miller, C., Peterson, D. E., Schubert, M. M., Spijkervet, F. K., and Horowitz, M. 2001. "Oral Mucositis and the Clinical and Economic Outcomes of Hematopoietic Stem-Cell Transplantation.” J. Clin. Oncol. 19 (8): 2201-5.

[4] Mallick, S., Benson, R., and Rath, G. K. "Radiation
Induced Oral Mucositis: A Review of Current Literature on Prevention and Management." Eur. Arch. Otorhinolaryngol. DOI: 10.1007//s00405-015-3964-6.

[5] Worthington, H. V., Clarkson, J. E., Bryan, G., Furness, S., Glenny, A. M., Littlewood, A., McCabe, M. G., Meyer, S., and Khalid, T. 2011. "Interventions for Preventing Oral Mucositis for Patients with Cancer Receiving Treatment." Cochrane Database Syst. Rev. 4: CD000978. DOI: 10.1002/14651858.CD000978.pub5.

[6] Mcguire, D. B., Fulton, J. S., Park, J., Brown, C. G., Correa, M. E., Eilers, J., and Elad, S. 2013. "Systematic Review of Basic Oral Care for the Management of Oral Mucositis in Cancer Patients." Support Care Cancer 21 (11): 3165-77.

[7] Birudaraj, R., Mahalingam, R., Li, X., and Jasti, B. R. 2005. "Advances in Buccal Drug Delivery. Critical Reviews in Therapeutic Drug Carrier Systems." 22(3): 295-330.

[8] Mehenert, W., and Mader, K. 2001. "Solid Lipid Nanoparticles: Production, Characterization and Applications." Advanced Drug Delivery Reviews 47: 165-196.

[9] Garud, A., Singh, D., and Garud, N. 2012. "Solid Lipid Nanoparticles (SLN): Method, Characterization and Applications." International Current Pharmaceutical Journal 1 (11): 384-93.

[10] Bissett, D. L. 2006. "Glucosamine: An Ingredient with Skin and Other Benefits." J. Cosmet. Dermatol. 5 (4): 309-15.

[11] Garner, S. T., Israel, B. J., Achmed, H., Capomacchia, A. C., Abney, T., and Azadi, P. 2007. "Transdermal permeability of N-acetyl-D-glucosamine." Pharm. Dev. Technol. 12 (2): 169-74.

[12] Laurent, T. C. 1970. "Structure of Hyaluronic Acid. Chemistry and the Molecular Biology of the Intracellular Matrix." London: Academic Press 703-32.

[13] Chen, W. Y. J., and Abatangelo, G. 1999. "Functions of Hyaluronan in Wound Repair." Wound Repair Reg. 7: 79-89.

[14] Brown, M. B., and Jones, S. A. 2005. "Hyaluronic Acid: A Unique Topical Vehicle for the Localized Delivery of Drugs to the Skin." JEADV 19: 308-18.

[15] Cirillo, N., Vicidomini, A., McCullough, M. J., Gambardella, A., Hassona, Y., Prime, S. S., and Colella, G. 2014. "A Hyaluronic Acid-Based Compound Unhibits Fibroblast Scenescence Induced by Oxidative Stress in vitro and Prevents Oral Mucositis in vivo." Journal Cellular Physiology DOI: 10.1002/jcp.24908.

[16] Favia, G., Mariggio, M. A., Maiorano, F, Cassano, A., Capodiferro, S., and Ribasti, D. 2008. "Accelerated Wound Healing Oral Soft Tissue and Angiogenic Effect Induced by a Pool of Aminoacids Combined to Sodium 
Hyaluronate (Aminogum)." J. Bio. Regul. Homeost. Agents 22: 109-16.

[17] Buchsel, P. C. 2008. "Polyvinylpyrrolidone-Sodium Hyaluronate Gel (Gelclair): A Bioadherent Oral Gel for the Treatment of Oral Mucositis and Other Painful Oral Lesions.” Expert. Opin. Drug Metab. Toxicol. 4: 1449-54.

[18] Vettor, M., Bleve, M., Bruni, G., Mondelli, A., Secchi, G. F., and Pavanetto, F. 2012. "Preliminary Evaluation of Particle Systems Visualization on the Skin Surface by Scanning Electron Microscopy and Transparency Profilometry." Skin and Research Technology 18(1): 45-54;

[19] Bioadhesion-Pharmaceutical Bulletin 23. 2011. Lubrizol Corporation.

[20] Hagerstrom, H, and Edsman, K. 2001. "Interpretation of Mucoadhesive Properties of Polymer Gel Preparations Using a Tensile Strength Method.” Journal of Pharmacy and Pharmacology 53: 1589-99.

[21] Kharenko, E. A., Larionova, N. I., and Demina, N. B. 2008. "Mucoadhesive Drug Delivery Systems: Quantitative Assessment of Interaction between Synthetic and Natural Polymer Films and Mucosa." Pharmaceutical Chemistry Journal 42 (7): 17-24.

[22] OECD Guidelines for the Testing of Chemicals, Section 4. Test $n^{\circ}$ 439: In vitro Skin Irritation: Reconstructed Human Epidermis. DOI:
10.1787/20745788; 26 July 2013.

[23] Nguyen, D. H., Beuerman, R. W., De Wever, B., and Rosdy, M. 2003. "Three-Dimensional Construct of the Human Corneal Epithelium for in vitro Toxicology." Alternatives Toxicological method Edited by Salem, H., Katz, S. A. London: CRC Press, 147-59.

[24] Mossman, T. 1983. "Rapid Colorimetric Assay for Cellular Growth and Survival: Application to Proliferation and Cytotoxicity Assays." Journal of Immunological Methods 65: 55-63.

[25] Ghadiri, M., Fatemi, S., Vatanara, A., Doroud, D., Najafabadi, A. R., Darabi, M., and Rahimi, A. A. 2012. "Loading Hydrophilic Drug in Solid Lipid Media as Nanoparticles: Statistical Modeling of Entrapment Efficiency and Particle Size." International Journal of Pharmaceutics 424: 128-37.

[26] Bleve, M., Pavanetto, F., Capra, P., Bruni, G., Corsini, C., and Perugini, P. "Development of Topical Formulation Containing SLN Loaded with N-acetyl-glucosamine." Article in Press.

[27] Smart, J. D. 2005. "The Basics and Underlying Mechanisms of Mucoadhesion." Advanced Drug Delivery Reviews 57: 1556-68.

[28] Andrews, G. P., Laverty, T. P., and Jones, D. S. 2009. "Mucoadhesive Polymeric Platforms for Controlled Drug Delivery Systems" Eur. J. Pharm. Biopharm. 71: 505-18. 